\title{
Healthy and Pathological Brain Aging: From the Perspective of Oscillations, Functional Connectivity, and Signal Complexity
}

\author{
Ryouhei Ishii ${ }^{a}$ b Leonides Canuet ${ }^{c}$ Yasunori Aoki ${ }^{a, d}$ Masahiro Hata ${ }^{a}$ \\ Masao Iwase $^{a}$ Shunichiro Ikeda ${ }^{\mathrm{e}}$ Keiichiro Nishida ${ }^{\mathrm{e}}$ Manabu lkeda ${ }^{a}$ \\ ${ }^{a}$ Department of Psychiatry, Osaka University Graduate School of Medicine, Suita, Japan; ${ }^{\mathrm{b}}$ Department of Palliative \\ Care, Ashiya Municipal Hospital, Ashiya, Japan; ' Department of Cognitive, Social and Organizational Psychology, \\ La Laguna University, Tenerife, Spain; ${ }^{d}$ Department of Psychiatry, Nissay Hospital, Osaka, Japan; ${ }^{e}$ Department of \\ Psychiatry, Kansai Medical University, Moriguchi, Japan
}

\section{Keywords}

Aging · Cognition · Electroencephalography ·

Magnetoencephalography · Event-related potentials ·

Oscillatory activity $\cdot$ Functional connectivity $\cdot$ Signal

complexity · Biomarkers

\begin{abstract}
Healthy aging is associated with impairment in cognitive information processing. Several neuroimaging methods such as functional magnetic resonance imaging, positron emission tomography and near-infrared spectroscopy have been used to explore healthy and pathological aging by relying on hemodynamic or metabolic changes that occur in response to brain activity. Since electroencephalography (EEG) and magnetoencephalography (MEG) are able to measure neural activity directly with a high temporal resolution of milliseconds, these neurophysiological techniques are particularly important to investigate the dynamics of brain activity underlying neurocognitive aging. It is well known that age is a major risk factor for Alzheimer's disease (AD), and that synaptic dysfunction represents an early sign of this disease as-
\end{abstract}

\section{KARGER}

(c) 2018 S. Karger AG, Basel

E-Mail karger@karger.com

www.karger.com/nps sociated with hallmark neuropathological findings. However, the neurophysiological mechanisms underlying $A D$ are not fully elucidated. This review addresses healthy and pathological brain aging from a neurophysiological perspective, focusing on oscillatory activity changes during the resting state, event-related potentials and stimulus-induced oscillatory responses during cognitive or motor tasks, functional connectivity between brain regions, and changes in signal complexity. We also highlight the accumulating evidence on age-related EEG/MEG changes and biological markers of brain neurodegeneration, including genetic factors, structural abnormalities on magnetic resonance images, and the biochemical changes associated with $A \beta$ deposition and tau pathology.

(c) 2018 S. Karger AG, Basel

\section{Introduction}

Aging is a physiological process that affects all body tissues. In the brain, aging is mainly associated with a decline in attention, memory, and other cognitive functions 
as well as an overall delay in the different steps of cognitive information processing: (i) input and coding, (ii) central processing, and (iii) decision of response and output [1]. Since age is a major risk factor for Alzheimer disease (AD), issues related to pathological aging and its link to disease biomarkers will also be addressed. Several methods have been applied to explore brain physiological and pathological aging, including functional magnetic resonance imaging (fMRI), positron emission tomography (PET), near-infrared spectroscopy (NIRS), electroencephalography (EEG), and magnetoencephalography (MEG). While fMRI, PET, and NIRS measure hemodynamic or metabolic changes that occur in response to brain activity, neurophysiological techniques such as EEG and MEG measure neural activity directly by detecting postsynaptic potentials in the cerebral cortex $[2,3]$. In this review, we will address neurocognitive aging from a neurophysiological perspective, focusing on oscillatory activity changes during the resting state, event-related potentials and stimulus-induced oscillatory responses during cognitive or motor tasks, the organization of brain functional networks, and changes in signal complexity. The signal complexity of EEG reflects the irregularity of wave shape and dynamics, and is influenced by healthy aging and associated with brain disorders such as AD. A major objective was to highlight the value of EEG and MEG in understanding the physiological mechanisms associated with brain aging.

\section{EEG and MEG Techniques}

Ever since Berger [4] reported temporal changes in electric potentials measurable on the scalp in 1929, EEG has been used for a direct and noninvasive measurement of brain cortical activity (i.e., electric potentials), not only in clinical practice but also in other fields such as basic research and industry. Especially in Japan, where the proportion of the aging population in the society is increasing at an historically unprecedented rate, EEG is a tool commonly used in clinical practice for the diagnosis of neuropsychiatric diseases in elderly people [5]. Both EEG and MEG are noninvasive techniques without the emission of radiation or production of noise from strong magnetic fields that affect PET and fMRI, respectively. Furthermore, techniques that rely on blood flow to assess brain function commonly require up to 6-7 s to process neural activity. EEG and MEG, however, measure brain electromagnetic activity with a high temporal resolution of milliseconds. This property is par- ticularly important for investigating the dynamics of neural activity underlying cognitive processing and agerelated changes [2]. EEG measures the potential distribution caused by electric currents on the scalp and MEG is able to measure the small magnetic fields generated by these electric currents in cortical pyramidal neurons [2]. However, compared to EEG, MEG signals are less affected by the resistive properties of the skin, skull, and cerebral fluids. Therefore, MEG is associated with a more accurate source localization of brain activity [6]. Nevertheless, both EEG and MEG capture transientevoked responses as well as cortical oscillatory dynamics at rest and during cognitive or sensory-motor tasks. Thus, these neurophysiological techniques are essential for the study of age-related changes in healthy elderly people that can help improve the understanding of the mechanisms underlying pathological aging and the management of dementia.

\section{Aging and Cortical Oscillations}

\section{Aging and Resting-State Oscillations}

Most EEG/MEG studies have explored the effects of aging on brain activity during the resting state. Physiological aging has been characterized as a progressive change in brain wave frequency, power, morphology, and distribution during rest. The main findings include: (i) a marked reduction in the amplitude of alpha activity (8-13 $\mathrm{Hz}$ ), (ii) a slowing of the background activity (dominant alpha rhythm), and (iii) a global increase of delta $(1-4 \mathrm{~Hz})$ and theta $(4-8 \mathrm{~Hz})$ power $[7,8]$. A dominant alpha activity in posterior brain regions with a typical reactivity (i.e., blocking or attenuation) to opening the eyes is probably the most prominent electromagnetic change in the brain, and represents a distinctive feature of normal brain function in the waking state [9]. Because alpha activity enhancement occurs in the visual cortex when the eyes are closed, and the activity is suppressed by visual stimulation, alpha rhythm is proposed to represent a cortical "idling" that facilitates cortical activation by different stimuli. This includes the facilitation of sensorimotor signal-processing transmission and memory acquisition, as memories are stored in the brain cortex $[8,10,11]$. The generation of these alpha oscillations is linked to thalamus-cortical interactions [10].

Babiloni et al. [12], using low-resolution brain electromagnetic tomography (LORETA) analysis in a large sample of healthy elderly subjects and young individuals, confirmed that alpha rhythm in posterior cortical regions de- 
Table 1. Age-related changes in alpha source oscillations and alpha functional connectivity

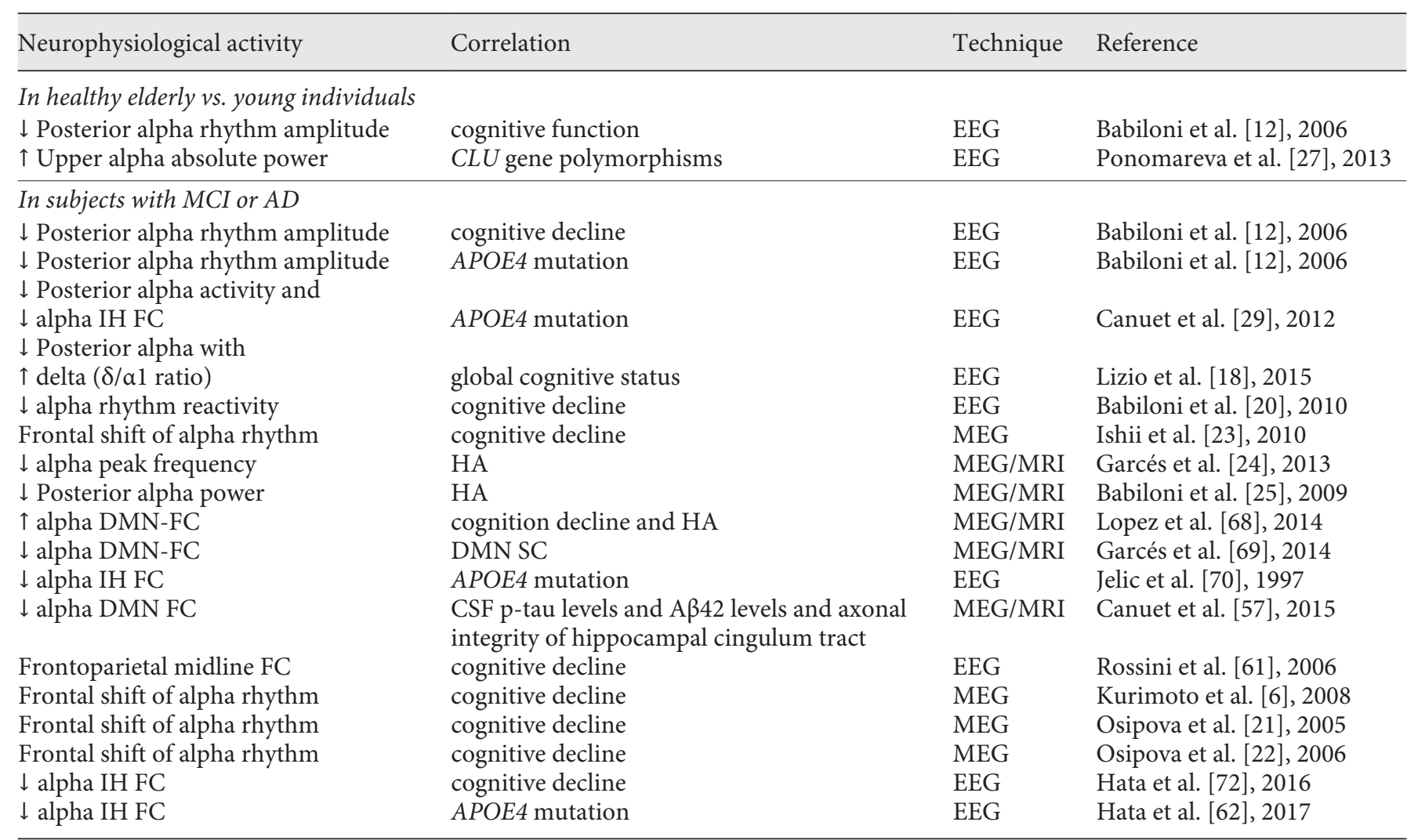

$\mathrm{AD}$, Alzheimer disease; DMN, default mode network; FC, functional connectivity; SC, structural connectivity; IH, interhemispheric; HA, hippocampal atrophy; MCI, mild cognitive impairment; EEG, electroencephalography; MEG, magnetoencephalography; MRI, magnetic resonance imaging.

creases in magnitude during physiological aging and correlates with the global cognitive level [13]. In alpha rhythm, 2 components can be identified. High or upper alpha $(10-13 \mathrm{~Hz})$ is a high-frequency component that reflects neural activity involved in the sense and elaboration of meaning-related information; the low-frequency component or lower alpha $(8-10 \mathrm{~Hz})$ is reported to be primarily associated with preparation or mental anticipation $[8$, $14]$. In the large-scale study of healthy volunteers performed by Babiloni et al. [12], an age-dependent decrease in posterior brain regions was found for both alpha components, indicating a relation between alpha activity and a decline in a wide range of cognitive functions with increasing age [14]. The decrease in occipital lobe alpha power with age is thought to be associated with functional changes in the cholinergic system of the basal forebrain that is considered important to maintain cortical excitatory activity, essential for the highest levels of cognitive function [15].

Brain Aging: Oscillations, Functional Connectivity, and Signal Complexity

\section{Alpha Oscillations and Cognition}

Oscillations in the alpha frequency are probably the activity the most affected by the aging process. Table 1 provides a list of relevant age-related changes in alpha sources and connectivity and the association with biomarkers. As mentioned above, one of the most established EEG findings concerning cortical oscillations in physiological aging is that alpha sources in posterior brain regions have significantly less magnitude in healthy elderly people than in young individuals [12]. Alpha source abnormalities can also be useful for discriminating cognitively normal elderly subjects from patients with cognitive impairment and dementia [16]. Recent evidence indicates that a decrease in alpha oscillations in the occipital areas during an eyes-closed resting-state, with a concomitant increase in delta current density, represents an EEG marker that provides a good classification rate (sensitivity $73.3 \%$, specificity $78 \%$, and accuracy $75.5 \%$ ) [17]. This EEG marker, defined as the parieto-occipital 
delta $(2-4 \mathrm{~Hz}) /$ low alpha $(8-10.5 \mathrm{~Hz})$ source ratio, has been associated with a lower global cognitive status and more abnormalities of white-matter integrity and cerebrospinal fluid (CSF) volume in subgroups of patients with $\mathrm{AD}[18]$. Based on these results, it has been proposed that, cognitive and functional status being equal, patients with $\mathrm{AD}$ who are EEG-positive for this marker may show a neurophysiological susceptibility, which could be used to improve the diagnosis in clinical studies or to assess the efficacy of pharmacological interventions [18]. Findings in other EEG studies on mild cognitive impairment (MCI) indicate that alpha rhythm can also discriminate patients with a stable cognitive state from those with unstable global cognition [19]. Furthermore, alpha reactivity to eye-opening shows a progressive impairment from healthy elderly to mild $\mathrm{AD}$ that correlates with cognitive decline [20]. These findings together suggest that EEG alpha rhythm is strongly influenced by physiological and pathological aging (Table 1).

MEG has also contributed to relating cortical oscillations, particularly alpha frequency oscillations, to clinical and biological markers of pathological aging. For instance, Osipova et al. [21] applied minimum current estimates to spontaneous alpha activity recorded with MEG in AD and MCI patients and normal elderly controls during the eyes-closed session. They reported that $\mathrm{AD}$ patients had predominant lower alpha band sources in the temporal regions whereas robust alpha sources were found near the parieto-occipital sulcus in the controls and MCI patients. They also pointed out that activation within the parieto-occipital region was significantly weaker and that in the right temporal area significantly enhanced in the AD patients [22]. These results suggest an increased temporal-lobe contribution coinciding with parieto-occipital deficits. They speculated that the slowing of spontaneous alpha oscillations and an anterior shift of a source of alpha activity of $\mathrm{AD}$ might not be associated with the involvement of a new set of oscillators, but with a gradual shift in the location and frequency of existing sources. We also found a significant increase in alpha oscillations in frontal regions, mainly over the prefrontal cortex, in patients with early-stage $\mathrm{AD}$ relative to healthy elderly controls, suggestive of a frontal shift of the posterior dominant alpha rhythm in these patients [23]. Additionally, the negative correlation between this alpha band abnormality and cognitive status demonstrates an association of a frontal shift of the posterior alpha activity with cognitive impairment in AD [23]. These findings suggest that a resting eyes-open/eyesclosed paradigm may elicit changes in alpha oscillatory power as new candidate physiological markers for this disorder.

Patients with early $\mathrm{AD}$ are reported to show a decrease in the peak frequency of occipital dominant alpha rhythm relative to healthy elderly subjects on MEG recordings. This abnormality showed a correlation with hippocampal volume, a structural marker of the disease [24]. EEG findings also support a link between alterations in alpha activity (i.e., decreased alpha power) and hippocampal atrophy (HA) [25] as well as correlations of upper/ low alpha power ratio changes with medial temporal cortical atrophy and memory performance [26].

A different line of research has focused on genotypic profiles and EEG neurophysiological markers in brain aging. Recent evidence suggests that genetic factors of pathological aging like clusterin (CLU) gene polymorphisms and the apolipoprotein E $\varepsilon 4$ (APOE4) mutation may affect upper alpha absolute power in nondemented elderly individuals [27], or reduce the amplitude of the posteriordominant alpha rhythm in early $\mathrm{AD}[28,29]$. Taken together, these alpha frequency findings suggest that the genotype is associated with age-related preclinical dysregulation of hippocampal function and contributes to $\mathrm{AD}$ pathophysiology. In addition to alpha power, alpha phase dynamics also show an association with age-related cognitive decline. This is supported by evidence of a correlation between the lower alpha phase resetting prior to the presentation of visual relevant stimuli and worse visual working memory performance in elderly subjects [30]. Overall, EEG alpha sources are thought to gradually deteriorate across time as cognitive function declines in healthy elderly individuals to clinically manifest AD [31].

\section{Delta/Theta Oscillations and Cognition}

A slowing of EEG/ MEG background activity is a neurophysiological sign commonly associated with healthy aging [32]. In cognitively normal elderly subjects, CSF total-tau and p-tau levels as well as the combined p-tau/ $A \beta 42$ ratio are associated with relative EEG theta power, especially in posterior brain regions. This increase in theta power correlates with a slowing of cognitive speed, indicating that increased theta oscillations in posterior regions may represent an early sign of neurodegeneration in the brain [33]. This activity is often interpreted as a slowing of alpha rhythm, In line with this view, the results of the study by Finnigan and Robertson [34], on healthy elderly subjects, proposed the existence of 2 forms of theta frequency oscillation, one indicative of healthy neurocognitive function and the other representing an EEG/ alpha slowing linked to future substantial cognitive de-
$4 \quad$ Neuropsychobiology DOI: $10.1159 / 000486870$
Ishii/Canuet/Aoki/Hata/Iwase/Ikeda/ Nishida/Ikeda 
cline. Although there is some consensus about the existence of age-related decreases in alpha oscillations, there are conflicting findings concerning the association between slow waves and healthy aging. Hartikainen et al. [35], using EEG, were among the first researchers to notice that delta and theta oscillations were lower in elderly people than in a group of younger subjects, leading to the assumption that a slow activity pattern is not always associated with aging. Furthermore, several MEG investigations have found a link between healthy aging and a global decrease in resting-state slow-wave power that may be accompanied by a worse performance on various cognitive tasks $[36,37]$. Interestingly, the healthy elderly subjects who were able to maintain enhanced delta and theta power, specifically in the temporal and central regions, exhibited improved performance of tasks, and was associated with perceptual speed and working memory functioning [36]. Taken together, these findings and those of Finnigan and Robertson [34] suggest that, when there is no alpha slowing, a relative increase in the power of slow activity, particularly theta oscillations, may be indicative of healthy neurocognitive function rather than of age-related cognitive decline [34-37].

In patients with prodromal $\mathrm{AD}$, the slowing of cortical activity in posterior brain regions appears to occur mainly in the delta band. Evidence for this argument is provided by Fernandez et al. [38] in an MEG study that reported increased occipital delta current density in individuals with prodromal $\mathrm{AD}$, with this activity likely reflecting dysfunctional synaptic transmission. In patients who have already progressed to full clinical $\mathrm{AD}$, an increase in both delta and theta oscillations was found in the temporoparietal regions, and this correlated with HA [39]. Babiloni et al. [40] also noted that the amplitude of delta EEG sources show correlation with white-matter lobar brain volume in prodromal and clinical AD. It seems that aging also induces delta abnormalities in oddball paradigms. In particular, delta event-related responses decrease with age, specifically across frontoparietal regions during visual oddball tasks [41]. Aging is also associated with other frequency-specific changes in oscillatory activity during oddball tasks, including a P300-related enhancement in theta oscillations in medial frontoparietal areas, along with increased prefrontal alpha activity in healthy middle-aged subjects compared to younger individuals [42]. This theta enhancement is absent in patients with AD during auditory oddball processing [43]. In addition to stimulus-induced oscillatory activity (i.e., phase-unlocked responses), aging is also associated with event-related potentials or stimulus phase-

Brain Aging: Oscillations, Functional Connectivity, and Signal Complexity locked activity, including a decline in P300 amplitude with increasing age [44]. A study performed by Kopp et al. [45], looking at age-related changes in the dual mechanisms of cognitive control, found that elderly subjects maintain good performance in task-switching at the expense of slower response times. P300 event-related potential abnormalities showed an age-related neural underrecruitment for proactive cognitive control (associated with brain activity for anticipatory task preparation) and neural overrecruitment for reactive cognitive control (associated with reduced preparatory activity but increased activation during task execution). Since P300 and related oscillations also reflect attention and memory updating processing [46], stimulus phase-locked and unlocked responses during oddball tasks can reveal abnormalities in several aspects of cognitive processing associated with aging.

Fontal midline theta rhythm is a distinct theta activity generated in the medial prefrontal cortex during focused attention [47]. This activity in the theta frequency band is reportedly affected by a healthy aging process. EEG recordings during visual attention tasks have revealed that, compared with young individuals, elderly people exhibit reduced frontal midline theta activity, which predicts the efficient maintenance of working memory [48]. These results suggest that brain aging alters the cortical circuitries of theta dynamics, leading to impairments in focused attention and working memory processing [46]. Others studies relate a decrease in frontal midline theta waves to abnormalities in reinforcement learning in healthy elderly [49]. This indicates that abnormalities in frontal theta oscillations underlie the neurophysiological mechanisms associated with neurocognitive aging.

\section{Beta Oscillations and Cognitive/Motor Function}

Age-related changes in beta frequency band at rest are not commonly reported. Beta oscillations are typically associated with movement-related activity, including movement observation, imagery, and execution, rather than with neurocognitive function [50]. Increasing age has been linked to greater baseline beta power in the motor cortex contralateral to the active hand. During the execution of movement, healthy aging has been associated with an increase in movement-related beta attenuation or desynchronization in the ipsilateral motor cortex, and a decrease in frequency in the motor cortex bilaterally [51]. These changes support the notion of enhanced motor cortex GABAergic inhibitory activity in elderly subjects. There are reports indicating that beta power in occipital regions is related to attentional modulation in the visual 
system [52]. Based on this finding, a decrease in beta band cortical activation during attentional modulation of visual motion processing in elderly people likely reflects age-related alertness deficits [53]. In pathological aging, a decrease in beta power over frontocentral regions relative to healthy elderly controls appeared to underlie visual working memory dysfunction, as revealed by MEG data during the Sternberg memory task [54]. These findings indicate that beta oscillations are not only associated with motor activities but also with modulation in the visual system.

There is evidence that, in addition to movement-related beta activity, aging also affects alpha sensorimotor oscillations in a different way. In a go/no go task, healthy elderly subjects showed greater beta rhythm attenuation during movement preparation and execution relative to young subjects in the sensorimotor cortex [55]. This was apparently an unexpected finding, as beta band desynchronization or attenuation during movement is considered an index of normal brain function $[11,56]$. This activity associated with a weaker beta rebound after movement, suggesting that the greater beta activity attenuation in elderly subjects during both response execution and preparation can be attributed to an enhanced investment of neural resources or neural overrecruitment in healthy aging [55]. Interestingly, an additional alpha rebound was observed only in elderly subjects after response inhibition. The overall findings speak in favor of different neural trajectories for sensorimotor alpha and beta oscillations in physiological aging.

\section{Oscillation-Based Connectivity}

Pathological aging is characterized by early synaptic disruption and synaptic loss that lead to white-matter abnormalities, anatomofunctional connectivity deficits and progressive cognitive decline $[29,57-60]$. This is known to be related to the magnitude and spatial distribution of intracellular aggregates of tau protein filaments (neurofibrillary tangles) and the extracellular deposition of $A \beta$ peptides (amyloid plaques) $[29,57]$. Thus, recent studies have looked at neuronal network organization in cognitively normal elderly subjects and in patients with $\mathrm{AD}$, and its association with disease biomarkers. In addition to abnormalities in alpha source oscillatory activity, disruption in functional connectivity in the alpha band is reported in biomarker-positive elderly subjects who progress to $\mathrm{AD}$ and in patients who have developed the disease [57, 60-62]. Rossini et al. [61] reported the chang- es of cortical connectivity and LORETA sources of EEG rhythms between the baseline (i.e., at the time of MCI diagnosis) and follow-up (approx. 14 months later) of MCI patients. They found stronger frontoparietal midline coherence as well as delta (temporal), theta (parietal, occipital, and temporal), and alpha 1 (central, parietal, occipital, temporal, and limbic) sources in MCI subjects than in stable subjects. They also showed that a low midline coherence and a weak temporal source were associated with a $10 \%$ annual rate of $\mathrm{AD}$ conversion, and this rate increased to 40 and $60 \%$ when a strong temporal delta source and a high midline gamma coherence were observed, respectively.

We also assessed EEG lagged-phase synchronization (LPS) in 28 probable AD patients and 30 healthy controls, as a measure of brain functional connectivity using eLORETA. We found that patients with AD showed significantly decreased LPS between most cortical regions in the delta band, and between the right dorsolateral prefrontal cortex and right posterior-inferior parietal lobule in the theta band relative to controls. In addition, some connections in the delta band were found to be associated with cognitive function, as measured by the MMSE. We suggested that $\mathrm{AD}$ and healthy elderly patients may have different patterns of LPS [62].

Graph theory analysis is a mathematical approach to quantify the topological organization and properties of human brain networks. A graph consisting of a set of nodes and edges is characterized by several metrics, and allows for the performance of spectral analysis. Among the most important graph metrics are the clustering coefficient of the nodes, a measure of local network connectivity, and the shortest path length between nodes which reflects global network efficiency. These metrics determine the type of network (i.e., ordered, small-world, or random), with the network combining high clustering and short path lengths that represents a small-world network [63]. The application of graph theory to EEG has revealed that the brain network in healthy elderly is characterized by a greater small-world network, as an index of normal network architecture, specifically in the alpha band, and a lesser small-world structure in faster-frequency bands relative to patients with $\mathrm{AD}$ [64]. In pathological aging, on the other hand, there is an early disruption of the network architecture. Hence, patients with AD show an inefficient, random type of network, or a loss of small-world network characteristics [65]. Studies on healthy subjects provide evidence that aging processes modulate brain functional networks in the upper alpha frequency band. In particular, the alpha-band characteristic path length, a measure of the
$6 \quad$ Neuropsychobiology DOI: $10.1159 / 000486870$
Ishii/Canuet/Aoki/Hata/Iwase/Ikeda/ Nishida/Ikeda 
efficiency of information processing in the network, shows a significant decrease with increasing age [66]. In the case of patients with prodromal $\mathrm{AD}$, the network path length decreases in the theta frequency band relative to healthy elderly subjects [67].

MEG spectral functional connectivity analysis at source level has shed light on the impairment in neuronal communication between cortical regions in pathological aging and its relation to disease biomarkers. An increasing number of studies report a pattern of hypersynchronization, specifically in the alpha frequency band, between certain brain regions in MCI or prodromal AD which is associated with cognitive decline. This increase in alpha synchronization mainly affects the temporal cortex and regions of the default mode network (DMN) [68]. This network is active during the resting state and is temporally interrupted or attenuated during the performance of cognitive tasks. Thus, the intrinsic network organization at rest allows the brain to allocate resources and ready itself for changes in response to stimuli [29]. An increase in DMN alpha functional connectivity in patients with MCI shows a correlation with HA and entorhinal atrophy [68]. A hypersynchronization pattern in MCI patients was also found in a recent blinded, multicenter MEG study. This functional connectivity abnormality was interpreted as an early sign of synaptic disruption, and a possible preclinical biomarker for early stage $\mathrm{AD}$ before a global cortical disconnection emerged with the progression of the disease [60]. Others have reported a decreased alpha functional connectivity within DMN regions in patients with MCI. This connectivity disruption was associated with DMN white-matter structural disconnection, as indicated by decreased fractional anisotropy along network tracts [69]. The discrepancies between studies on functional connectivity in MCI may be due, at least in part, to the lack of markers of pathological disease. The studies may have included a heterogeneous sample of patients with cognitive deficits and not only those who would progress to AD type of dementia. A diagnosis based on tau protein and $\mathrm{A} \beta$ biomarkers can help elucidate this problem.

With regard to genetic markers, APOE4 has been related to a decrease in EEG alpha interhemispheric connectivity in frontal and parietotemporal regions as assessed by LPS [29] and linear coherence [70]. The influence of $A P O E 4$ on aging and neurodegeneration has also been explored with MEG, reporting decreased long-range functional connectivity in slow frequencies in APOE4 carriers, where frontal and temporal connections were particularly compromised [71].

Brain Aging: Oscillations, Functional Connectivity, and Signal Complexity
In the search for histopathological biomarkers of aging and their effects on brain activity, resting-state MEG has been used to assess whether synaptic dysfunction and structural connectivity disruption associate with CSF biomarkers, in particular $\mathrm{p}$-tau and $\mathrm{A} \beta$ levels in patients with prodromal AD. It was found that markers of amyloid deposition and neuronal injury are related to a dual pattern of cortical network disruption in the alpha and higherfrequency bands, affecting key regions of the DMN (e.g., the anterior and posterior cingulate cortex), the orbitofrontal cortex, and the temporal lobe regions. Interestingly, p-tau-related DMN alpha connectivity deficits were found to be associated with structural connectivity abnormalities, particularly with impaired axonal integrity of the hippocampal cingulum. Overall connectivity abnormalities predicted cognitive impairment [57]. These findings demonstrate that DMN functional and structural connectivity deficits are at the center of neurocognitive aging and neurodegeneration, and underlie AD pathology. We examined the association between CSF biomarkers and EEG parameters in 14 probable $\mathrm{AD}$ patients by using eLORETA LPS, and assessed the correlations between CSF biomarkers and EEG parameters. We found that patients with $\mathrm{AD}$ showed a significant negative correlation between CSF A $\beta 42$ concentration and the logarithms of current source density across the right temporal area in the theta band. Total tau concentration was negatively correlated with the LPS between the left frontal eye field and the right auditory area in the alpha- 2 band in patients with $\mathrm{AD}$ [72]. Our results suggest that $\mathrm{AD}$ biomarkers, in particular CSF A $\beta 42$ and total tau concentrations, are associated with the EEG parameters, current source density and LPS, respectively.

\section{EEG Signal Complexity and Aging}

The signal complexity of EEG, a reflection of the irregularity of the wave shape and dynamics, is reported to be influenced by healthy aging and associated with brain disorders such as $\mathrm{AD}[73,74]$. Studies using fractal dimension demonstrated that resting-state EEG signal complexity increases from youth to maturity, followed by a reduction in healthy elderly. These studies also provided evidence indicating that, in $\mathrm{AD}$, the signal complexity is further reduced as cognitive function declines [73, 74].

To elucidate the physiological mechanisms of brain aging, we divided healthy subjects into 3 age groups: young (age 17-29 years, $n=24)$, middle-aged (30-59 years, $n=14)$ and elderly $(60-77$ years, $n=11)$. and used 


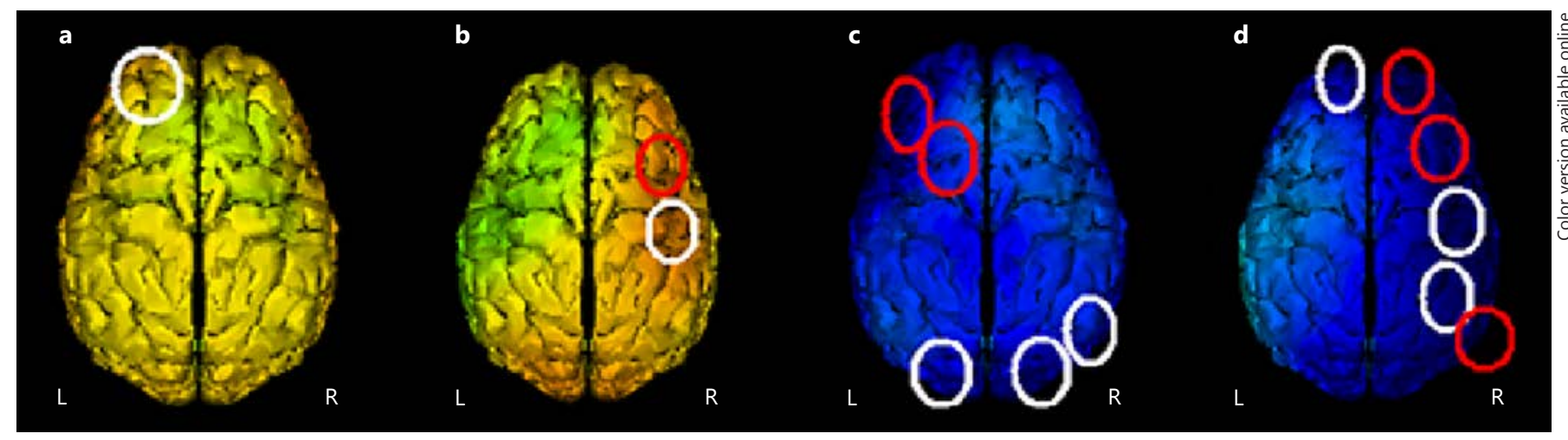

Fig. 1. EEG distribution of significantly different normalized power variance (an index of signal complexity) across age groups for theta and beta frequencies. a, b Differences between the middleaged and young groups (a: theta range in the left frontopolar area, Fp1 site; $p=0.038$. b: beta range in the right dorsolateral prefrontal cortex, F4 site; $p<0.01$, C4 site $p=0.020)$. c, d Differences between the elderly and middle-aged groups (c: theta range in the left prefrontal cortex, F3 site; $p<0.01$, F7 site; $p<0.01$, temporo-occipital areas, T6 site; $p=0.019, \mathrm{O} 1$ site; $p=0.042$, O2 site; $p=0.023$. d: beta range in the left frontopolar cortex and right hemispheric areas, Fp1 site; $p=0.049$, Fp2 site; $p<0.01$, F4 site; $p<0.01, \mathrm{C} 4$ site; $p=0.012, \mathrm{P} 4$ site $p=0.015$, T6 site; $p<0.01)$. White circles indicate $p<0.05$; red circles, $p<0.01$. L, left; $\mathrm{R}$, right. neural activity topography (NAT) analysis to assess agerelated changes in brain wave power variance [75]. NAT is a novel analysis method that measures EEG normalized power variance (NPV), an index of waveform complexity [76]. This method has been used successfully to detect changes in the complexity of signal dynamics associated with cognitive and clinical improvement in patients with normal pressure hydrocephalus who respond to shunt operation [77]. It was also reported that an NPV decrease represents the stabilization of cortical electrical activity which reflects functional improvement, while an NPV increase represents the destabilization of cortical electrical activity which reflects functional worsening [77]. In our study, NAT results showed an increase in NPV values from younger to middle-aged subjects, and a decrease in the values from middle-aged to the elderly, after an analysis of all regions and locations. Age-related abnormalities were observed mainly in the theta activity across the left prefrontal regions involved in working memory and executive functions as well as in beta activity, mainly over the broad areas of the right hemisphere (Fig. 1) [75]. The existence of age-related changes in the beta band, associated with attentional and motor functions and predominantly in right hemispheric areas, is consistent with the right-hemisphere-aging model proposed by Brown and Jaffe [78]. These findings using NAT suggest that signal complexity declines with age after adulthood and provide support for previous EEG studies on healthy aging using fractal dimension [76].
NAT analysis may be a useful tool for the assessment of the association between neurocognitive aging and changes in brain signal complexity.

\section{Conclusions}

This review focused on the influence of healthy aging on several neurophysiological measures, derived from EEG and MEG data, and ranging from cortical oscillatory activity to network organization and signal complexity dynamics. Since age is a major risk factor for AD, the influence of pathological aging on brain activity was addressed as well. An interesting approach in this study lies in the link between the age-related neurophysiological findings and biological markers of brain neurodegeneration, including genetic factors (i.e., CLU and the APOE4 mutation), MRI structural abnormalities (i.e., HA), and biochemical changes associated with $\mathrm{A} \beta$ deposition and tau pathology. Compared with young adults, elderly people commonly show an EEG/MEG decrease in alpha oscillatory activity and alpha rhythm reactivity as well as a slowing of the background activity, with an increase in delta or theta power diffusely or in posterior region rhythm abnormalities. Slow activity, however, is sometimes found to be reduced in healthy elderly subjects compared to in young individuals, and this may be linked to a relatively poor cognitive performance. It seems that when there is no concomitant alpha slowing, a relative 
increase in power of slow activity, particularly theta oscillations, may be indicative of healthy neurocognitive function rather than of age-related cognitive decline.

Healthy and pathological aging also affect the cortical oscillations underlying various cognitive processes, sensory-motor activities, and P300 abnormalities, such as stimulus phase-locked activity and stimulus-induced oscillations. Consistent with the notion that synaptic dysfunction and abnormal neuronal communication is critical to $\mathrm{AD}$, growing evidence indicates that aging substantially influences the organization of brain networks, as measured by oscillation-based functional connectivity and graph theory analysis. An interhemispheric hypersynchronization pattern in the alpha band appears to play an important role in prodromal $\mathrm{AD}$ pathophysiology. Overall, the literature reviewed showed that EEG and MEG techniques and analyses have evolved to capture the brain activity that underlies healthy neurocognitive aging, to facilitate the early detection of brain neuropathology markers for $\mathrm{AD}$ diagnosis and progression, at least at a group level. Future studies may help to obtain neurophysiological markers of the disease that can be useful for individual subject diagnosis and treatment management.

\section{Disclosure Statement}

The authors declare that they have no competing interests.

\section{Author Contributions}

R.I., L.C., S.I., Y.A., and M.H. analyzed and interpreted the EEG data of normal controls. R.I., M.I., and M.I. performed the major contributions in the writing of the paper. All authors read and approved the final manuscript.

\section{References}

1 Birren JE, Fisher LM: Aging and speed of behavior: possible consequences for psychological functioning. Annu Rev Psychol 1995;46: 329-353.

2 Aine CJ: A conceptual overview and critique of functional neuroimaging techniques in humans. I. MRI/FMRI and PET. Crit Rev Neurobiol 1995;9:229-309.

3 Hari R, Parkkonen L: The brain timewise: how timing shapes and supports brain function. Philos Trans R Soc Lond B Biol Sci 2015; 370:1668.

4 Berger H: Über das Elektroenkephalogramm des Menschen. Arch Psychiatr Nervenkr 1929;87:527-570.

5 Nishida K, Yoshimura M, Isotani T, Yoshida T, Kitaura Y, Saito A, et al: Differences in quantitative EEG between frontotemporal dementia and Alzheimer's disease as revealed by LORETA. Clin Neurophysiol 2011;122: 1718-1725.

-6 Kurimoto R, Ishii R, Canuet L, Ikezawa K, Azechi M, Iwase M, et al: Event-related synchronization of alpha activity in early $\mathrm{Al}$ zheimer's disease and mild cognitive impairment: an MEG study combining beamformer and group comparison. Neurosci Lett 2008; 443:86-89.

7 Klass DW, Brenner RP: Electroencephalography of the elderly. J Clin Neurophysiol 1995; 12:116-131.

8 Klimesch W: EEG alpha and theta oscillations reflect cognitive and memory performance: a review and analysis. Brain Res Brain Res Rev 1999;29:169-195.

-9 Ikezawa K, Ishii R, Iwase M, Kurimoto R, Canuet L, Takahashi H, et al: Decreased alpha event-related synchronization in the left pos- terior temporal cortex in schizophrenia: a magnetoencephalography-beamformer study. Neurosci Res 2011;71:235-243.

10 Pfurtscheller G: Event-related synchronization (ERS): an electrophysiological correlate of cortical areas at rest. Electroencephalogr Clin Neurophysiol 1992;83:62-69.

-11 Pfurtscheller G, Lopes da Silva FH: Event-related EEG/MEG synchronization and desynchronization: basic principles. Clin Neurophysiol 1999;110:1842-1857.

12 Babiloni C, Binetti G, Cassarino A, Dal Forno G, Del Percio C, Ferreri F, et al: Sources of cortical rhythms in adults during physiological aging: a multicentric EEG study. Hum Brain Mapp 2006;27:162-172.

13 Babiloni C, Binetti G, Cassetta E, Dal Forno G, Del Percio C, Ferreri F, et al: Sources of cortical rhythms change as a function of cognitive impairment in pathological aging: a multicenter study. Clin Neurophysiol 2006; 117:252-268.

14 Klimesch W: EEG-alpha rhythms and memory processes. Int J Psychophysiol 1997;26: 319-340.

15 Sarter M, Bruno JP: Cortical acetylcholine, reality distortion, schizophrenia, and Lewy body dementia: too much or too little cortical acetylcholine? Brain Cogn 1998;38:297-316.

-16 Babiloni C, Visser PJ, Frisoni G, De Deyn PP, Bresciani L, Jelic V, et al: Cortical sources of resting EEG rhythms in mild cognitive impairment and subjective memory complaint. Neurobiol Aging 2010;31:1787-1798.

17 Babiloni C, Triggiani AI, Lizio R, Cordone S, Tattoli G, Bevilacqua V, et al: Classification of single normal and Alzheimer's disease individuals from cortical sources of resting state
EEG rhythms. Front Neurosci 2016;10:47.

18 Lizio R, Del Percio C, Marzano N, Soricelli A, Yener GG, Başar E, et al: Neurophysiological assessment of Alzheimer's disease individuals by a single electroencephalographic marker. J Alzheimers Dis 2015;49:159-177.

19 Babiloni C, Frisoni GB, Vecchio F, Lizio R, Pievani M, Cristina G, et al: Stability of clinical condition in mild cognitive impairment is related to cortical sources of alpha rhythms: an electroencephalographic study. Hum Brain Mapp 2011;32:1916-1931.

20 Babiloni C, Lizio R, Vecchio F, Frisoni GB, Pievani M, Geroldi C, et al: Reactivity of cortical alpha rhythms to eye opening in mild cognitive impairment and Alzheimer's disease: an EEG study. J Alzheimers Dis 2010;22: 1047-1064.

21 Osipova D, Ahveninen J, Jensen O, Ylikoski A, Pekkonen E: Altered generation of spontaneous oscillations in Alzheimer's disease. Neuroimage 2005;27:835-841.

-22 Osipova D, Rantanen K, Ahveninen J, Ylikoski R, Häppölä O, Strandberg T, et al: Source estimation of spontaneous MEG oscillations in mild cognitive impairment. Neurosci Lett 2006;405:57-61.

23 Ishii R, Canuet L, Kurimoto R, Ikezawa K, Aoki Y, Azechi M, et al: Frontal shift of posterior alpha activity is correlated with cognitive impairment in early Alzheimer's disease: a magnetoencephalography-beamformer study. Psychogeriatrics 2010;10:138-143.

24 Garcés P, Vicente R, Wibral M, Pineda-Pardo JÁ, López ME, Aurtenetxe S, et al: Brain-wide slowing of spontaneous alpha rhythms in mild cognitive impairment. Front Aging Neurosci 2013;5:100. 
-25 Babiloni C, Frisoni GB, Pievani M, Vecchio F, Lizio R, Buttiglione M, et al: Hippocampal volume and cortical sources of EEG alpha rhythms in mild cognitive impairment and Alzheimer disease. Neuroimage 2009;44:123135.

-26 Moretti DV, Paternicò D, Binetti G, Zanetti O, Frisoni GB: EEG upper/low alpha frequency power ratio relates to temporo-parietal brain atrophy and memory performances in mild cognitive impairment. Front Aging Neurosci 2013;5:63.

27 Ponomareva N, Andreeva T, Protasova M, Shagam L, Malina D, et al: Age-dependent effect of Alzheimer's risk variant of CLU on EEG alpha rhythm in non-demented adults. Front Aging Neurosci 2013;5:86

-28 Babiloni C, Benussi L, Binetti G, Cassetta E, Dal Forno G, Del Percio C, et al: Apolipoprotein $\mathrm{E}$ and alpha brain rhythms in mild cognitive impairment: a multicentric electroencephalogram study. Ann Neurol 2006;59: 323-334.

-29 Canuet L, Tellado I, Couceiro V, Fraile C, Fernandez-Novoa L, Ishii R, et al: Resting-state network disruption and ApoE genotype in $\mathrm{Al}$ zheimer's disease: a lagged functional connectivity study. PLoS One 2012;7:e46289.

-30 Tran TT, Hoffner NC, LaHue SC, Tseng L, Voytek B: Alpha-phase dynamics predict agerelated visual working memory decline. Neuroimage 2016;143:196-203.

-31 Babiloni C, Del Percio C, Lizio R, Marzano N, Infarinato F, Soricelli A, et al: Cortical sources of resting state electroencephalographic alpha rhythms deteriorate across time in subjects with amnesic mild cognitive impairment. Neurobiol Aging 2014;35:130-142.

- 32 Rossini PM, Rossi S, Babiloni C, Polich J: Clinical neurophysiology of aging brain: from normal aging to neurodegeneration. Prog Neurobiol 2007;83:375-400.

- 33 Stomrud E, Hansson O, Minthon L, Blennow K, Rosén I, Londos E: Slowing of EEG correlates with CSF biomarkers and reduced cognitive speed in elderly with normal cognition over 4 years. Neurobiol Aging 2010;31:215223.

-34 Finnigan S, Robertson IH: Resting EEG theta power correlates with cognitive performance in healthy older adults. Psychophysiology 2011;48:1083-1087.

-35 Hartikainen P, Soininen H, Partanen J: Aging and spectral analysis of EEG in normal subjects: a link to memoryand CSF AChE. Acta Neurol Scand 1992;86:148-155.

36 Vlahou EL, Thurm F, Kolassa IT, Schlee W: Resting-state slow wave power, healthy aging and cognitive performance. Sci Rep 2014;4: 5101.

- 37 Leirer VM, Wienbruch C, Kolassa S, Schlee W, Elbert T, Kolassa IT: Changes in cortical slow wave activity in healthy aging. Brain Imaging Behav 2011;5:222-228.

-38 Fernandez A, Turrero A, Zuluaga P, Gil-Gregorio P, del Pozo F, Maestu F, et al: MEG delta mapping along the healthy aging-Alzheim- er's disease continuum: diagnostic implications. J Alzheimers Dis 2013;35:495-507.

39 Fernández A, Arrazola J, Maestú F, Amo C, Gil-Gregorio P, Wienbruch C, et al: Correlations of hippocampal atrophy and focal lowfrequency magnetic activity in Alzheimer disease: volumetric MR imaging-magnetoencephalographic study. AJNR Am J Neuroradiol 2003;24:481-487.

40 Babiloni C, Frisoni G, Steriade M, Bresciani L, Binetti G, Del Percio C, et al: Frontal white matter volume and delta EEG sources negatively correlate in awake subjects with mild cognitive impairment and Alzheimer's disease. Clin Neurophysiol 2006;117:11131129.

- 41 Emek-Savaș DD, Güntekin B, Yener GG, Başar E: Decrease of delta oscillatory responses is associated with increased age in healthy elderly. Int J Psychophysiol 2016;103:103109.

42 Yordanova JY, Kolev VN, Başar E: EEG theta and frontal alpha oscillations during auditory processing change with aging. Electroencephalogr Clin Neurophysiol 1998;108:497-505.

43 Caravaglios G, Castro G, Costanzo E, Di Maria G, Mancuso D, Muscoso EG: $\theta$ Power responses in mild Alzheimer's disease during an auditory oddball paradigm: lack of theta enhancement during stimulus processing. J Neural Transm 2010;117:1195-1208.

44 van Dinteren R, Arns M, Jongsma ML, Kessels RP: Combined frontal and parietal P300 amplitudes indicate compensated cognitive processing across the lifespan. Front Aging Neurosci 2014;6:294.

45 Kopp B, Lange F, Howe J, Wessel K: Age-related changes in neural recruitment for cognitive control. Brain Cogn 2014;85:209-219.

46 Ishii R, Canuet L, Herdman A, Gunji A, Iwase $\mathrm{M}$, Takahashi $\mathrm{H}$, et al: Cortical oscillatory power changes during auditory oddball task revealed by spatially filtered magnetoencephalography. Clin Neurophysiol 2009;120:497504.

47 Ishii R, Shinosaki K, Ukai S, Inouye T, Ishihara T, Yoshimine T, et al: Medial prefrontal cortex generates frontal midline theta rhythm. Neuroreport 1999;10:675-679.

48 Kardos Z, Tóth B, Boha R, File B, Molnár M: Age-related changes of frontal-midline theta is predictive of efficient memory maintenance. Neuroscience 2014;273:152-162.

49 van de Vijver I, Cohen MX, Ridderinkhof KR: Aging affects medial but not anterior frontal learning-related theta oscillations. Neurobiol Aging 2014;35:692-704.

50 Honaga E, Ishii R, Kurimoto R, Canuet L, Ikezawa $\mathrm{K}$, Takahashi $\mathrm{H}$, et al: Post-movement beta rebound abnormality as indicator of mirror neuron system dysfunction in autistic spectrum disorder: an MEG study. Neurosci Lett 2010;478:141-145.

51 Rossiter HE, Davis EM, Clark EV, Boudrias $\mathrm{MH}$, Ward NS: Beta oscillations reflect changes in motor cortex inhibition in healthy ageing. Neuroimage 2014;91:360-365.
52 Gola M, Magnuski M, Szumska I, Wróbel A: EEG beta band activity is related to attention and attentional deficits in the visual performance of elderly subjects. Int J Psychophysiol 2013;89:334-341.

53 Gola M, Kamiński J, Brzezicka A, Wróbel A: $\beta$ band oscillations as a correlate of alertnesschanges in aging. Int J Psychophysiol 2012;85: 62-67.

54 Kurimoto R, Ishii R, Canuet L, Ikezawa K, Iwase M, Azechi M, et al: Induced oscillatory responses during the Sternberg's visual memory task in patients with Alzheimer's disease and mild cognitive impairment. Neuroimage 2012;59:4132-4140.

55 Schmiedt-Fehr C, Mathes B, Kedilaya S, Krauss J, Basar-Eroglu C: Aging differentially affects alpha and beta sensorimotor rhythms in a go/nogo task. Clin Neurophysiol 2016; 127:3234-3242.

56 Neuper C, Pfurtscheller G: Evidence for distinct beta resonance frequencies in human EEG related to specific sensorimotor cortical areas. Clin Neurophysiol 2001;112:20842097.

57 Canuet L, Pusil S, López ME, Bajo R, PinedaPardo JÁ, Cuesta P, et al: Network disruption and cerebrospinal fluid amyloid-beta and phospho-tau levels in mild cognitive impairment. J Neurosci 2015;35:10325-10330.

- 58 Rémy F, Vayssière N, Saint-Aubert L, Barbeau E, Pariente J: White matter disruption at the prodromal stage of Alzheimer's disease: relationships with hippocampal atrophy and episodic memory performance. Neuroimage Clin 2015;7:482-492.

59 Stam CJ, Jones BF, Manshanden I, van Cappellen van Walsum AM, Montez T, Verbunt JP, et al: Magnetoencephalographic evaluation of resting-state functional connectivity in Alzheimer's disease. Neuroimage 2006;32: 1335-1344.

60 Maestu F, Peña JM, Garcés P, González S, Bajo R, Bagic A, et al; Magnetoencephalography International Consortium of Alzheimer's Disease: A multicenter study of the early detection of synaptic dysfunction in mild cognitive impairment using magnetoencephalography-derived functional connectivity. Neuroimage Clin 2015;9:103-109.

-61 Rossini PM, Del Percio C, Pasqualetti P, Cassetta E, Binetti G, Dal Forno G, et al: Conversion from mild cognitive impairment to Alzheimer's disease is predicted by sources and coherence of brain electroencephalography rhythms. Neuroscience 2006;143:793-803.

62 Hata M, Tanaka T, Kazui H, Ishii R, Canuet L, Pascual-Marqui RD, et al: cerebrospinal fluid biomarkers of Alzheimer's disease correlate with electroencephalography parameters assessed by exact low-resolution electromagnetic tomography (eLORETA). Clin EEG Neurosci 2017;48:338-347.

$63 \mathrm{He}$ Y, Evans A: Graph theoretical modeling of brain connectivity. Curr Opin Neurol 2010; 23:341-350. 
64 Miraglia F, Vecchio F, Bramanti P, Rossini PM: EEG characteristics in "eyes-open" versus "eyes-closed" conditions: small-world network architecture in healthy aging and age-related brain degeneration. Clin Neurophysiol 2016;127:1261-1268.

- 65 Stam CJ, Jones BF, Nolte G, Breakspear M, Scheltens P: Small-world networks and functional connectivity in Alzheimer's disease. Cereb Cortex 2007;17:92-99.

66 Vecchio F, Miraglia F, Bramanti P, Rossini PM: Human brain networks in physiological aging: a graph theoretical analysis of cortical connectivity from EEG data. J Alzheimers Dis 2014;41:1239-1249.

-67 Vecchio F, Miraglia F, Marra C, Quaranta D, Vita MG, Bramanti P, et al: Human brain networks in cognitive decline: a graph theoretical analysis of cortical connectivity from EEG data. J Alzheimers Dis 2014;41:113-127.

68 Lopez ME, Bruña R, Aurtenetxe S, PinedaPardo JÁ, Marcos A, Arrazola J, et al: Alphaband hypersynchronization in progressive mild cognitive impairment: a magnetoencephalography study. J Neurosci 2014;34: 14551-14559.
69 Garcés P, Angel Pineda-Pardo J, Canuet L, Aurtenetxe S, López ME, Marcos A, et al: The default mode network is functionally and structurally disrupted in amnestic mild cognitive impairment - a bimodal MEG-DTI study. Neuroimage Clin 2014;6:214-221.

70 Jelic V, Julin P, Shigeta M, Nordberg A, Lannfelt L, Winblad B, et al: Apolipoprotein E ع 4 allele decreases functional connectivity in $\mathrm{Al}$ zheimer's disease as measured by EEG coherence. J Neurol Neurosurg Psychiatry 1997;63: 59-65.

71 Cuesta P, Garcés P, Castellanos NP, López ME, Aurtenetxe S, Bajo R, et al: Influence of the ApoE $\varepsilon 4$ allele and mild cognitive impairment diagnosis in the disruption of the MEG resting state functional connectivity in sources space. J Alzheimers Dis 2015;44:493-505.

72 Hata M, Kazui H, Tanaka T, Ishii R, Canuet L, Pascual-Marqui RD, et al: Functional connectivity assessed by resting state EEG correlates with cognitive decline of Alzheimer's disease - An eLORETA study. Clin Neurophysiol 2016;127:1269-1278.

73 Zappasodi F, Marzetti L, Olejarczyk E, Tecchio F, Pizzella V: Age-related changes in electroencephalographic signal complexity. PLoS One 2015;10:e0141995.
74 Smits FM, Porcaro C, Cottone C, Cancelli A, Rossini PM, Tecchio F: Electroencephalographic fractal dimension in healthy ageing and Alzheimer's disease. PLoS One 2016; 11:e0149587.

75 Ishii R, Aoki Y, Kurimoto R, Ikeda S, Hata M, Iwase $\mathrm{M}$, et al: Clinical neurophysiology of normal aging: a review of the literature. Japanese J Geriatric Psychiat 2012;23:420-428.

76 Musha T, Matsuzaki H, Kobayashi Y, Okamoto Y, Tanaka M, Asada T: EEG markers for characterizing anomalous activities of cerebral neurons in NAT (neuronal activity topography) method. IEEE Trans Biomed Eng 2013;60:2332-2338.

77 Aoki Y, Kazui H, Tanaka T, Ishii R, Wada T, Ikeda $\mathrm{S}$, et al: EEG and neuronal activity topography analysis can predict effectiveness of shunt operation in idiopathic normal pressure hydrocephalus patients. Neuroimage Clin 2013;3:522-530

78 Brown JW, Jaffe J: Hypothesis on cerebral dominance. Neuropsychologia 1975;13:107110 